\title{
Promoter Methylation Status of Two Novel Human Genes, UBE2Q1 and UBE2Q2, in Colorectal Cancer: a New Finding in Iranian Patients
}

\author{
Pooneh Mokarram ${ }^{1}$, Fatemeh Shakiba-Jam ${ }^{1}$, Soudabeh Kavousipour ${ }^{2}$, Mostafa \\ Moradi Sarabi ${ }^{1}$, Atefeh Seghatoleslam ${ }^{1 *}$
}

\begin{abstract}
Background: The ubiquitin-proteasome system (UPS) degrades a variety of proteins which attach to specific signals. The ubiquitination pathway facilitates degradation of damaged proteins and regulates growth and stress responses. This pathway is altered in various cancers, including acute lymphoblastic leukemia, head and neck squamous cell carcinoma and breast cancer. Recently it has been reported that expression of newly characterized human genes, UBE2Q1 and UBE2Q2, putative members of ubiquitin-conjugating enzyme family (E2), has been also changed in colorectal cancer. Epigenetics is one of the fastest-growing areas of science and nowadays has become a central issue in biological studies of diseases. According to the lack of information about the role of epigenetic changes on gene expression profiling of UBE2Q1 and UBE2Q2, and the presence of CpG islands in the promoter of these two human genes, we decided to evaluate the promoter methylation status of these genes as a first step. Materials and Methods: The promoter methylation status of UBE2Q1 and UBE2Q2 was studied by methylation-specific PCR (MSP) in tumor samples of 60 colorectal cancer patients compared to adjacent normal tissues and 20 non-malignant controls. The frequency of the methylation for each gene was analyzed by chi-square method. Results: MSP results revealed that UBE2Q2 gene promoter were more unmethylated, while a higher level of methylated allele was observed for UBE2Q1 in tumor tissues compared to the adjacent normal tissues and the non malignant controls. Conclusions: UBE2Q1 and UBE2Q2 genes show different methylation profiles in CRC cases.
\end{abstract}

Keywords: UBE2Q1 - UBE2Q2 - promoter methylation - colorectal cancer

Asian Pac J Cancer Prev, 16 (18), 8247-8252

\section{Introduction}

Ubiquitin-proteasome system (UPS) acts in many basic cellular processes such as cell cycle regulation, differentiation, proliferation (Zhu et al., 2013), apoptosis, gene transcription and many others by degradation of proteins (Bassermann and Pagano, 2010). The modification of proteins by ubiquitin is an important mechanism for targeting abnormal proteins for degradation. UPS is also involved in pathophysiological processes of many diseases including cancer (Zhu et al., 2013), ubiquitination is performed with cooperation of at least three classes of enzymes: ubiquitin-activating enzymes (E1), ubiquitinconjugating enzymes (E2), and ubiquitin-protein ligases (E3). UBE2Q1 and UBE2Q2 (previously known as LOC92912), as the newly characterized human genes are members of E2 ubiquitin-conjugating enzyme family (van Wijk and Timmers, 2010).

There are much evidences that deregulation of ubiquitin-proteasome pathway can lead to cancer development through the mechanisms that control the stability of regulating transcriptionand growth factors. We have previously showed that the expression profiling of UBE2Q1 and UBE2Q2 are altered in different malignancies (Seghatoleslam et al., 2006; Seghatoleslam et al, 2009; Nikseresht et al., 2010; Seghatoleslam et al., 2012; Shafiee et al., 2013; Seghatoleslam et al., 2014; Shafiee et al., 2014).

According to our recent publication, these two members of E2 family, UBE2Q1 and UBE2Q2, are over-expressed in Colorectal Cancer (CRC) patients and several colorectal cell lines (Shafiee et al 2013 and 2014). However, down regulation of UBE2Q1 has been reported to be occurred in Acute Lymphoblastic Leukemia (ALL) (Seghatoleslam et al., 2014).

Some members of E2 family of human ubiquitinconjugating enzymes have been also shown to be overexpressed in hypo - pharyngeal tumors (Voutsadakis, 2008), breast cancer (Chen and Madura, 2005), ALL (Cheng et al., 2006) and CRC (Chen et al., 2002) .

Furthermore, the up-regulation of UBE2Q1 has been also observed in breast cancer (Srivastava et al.,

${ }^{1}$ Department of Biochemistry, School of Medicine, ${ }^{2}$ School of Advanced Medical Science and Technologies, Shiraz University of Medical Sciences, Shiraz, Iran *For correspondence: seghatolea@sums.ac.ir 
2014) and the alteration of UBE2Q2 was also reported in fibroblastoma, glioma, recurrent head and neck and alveolar epithelial carcinoma (Maeda et al., 2009).

Several studies have shown that CRC is a multifactorial disease with the several genetic and epigenetic factors involved in this cancer initiation and progression. Furthermore, epigenetic analysis of tumor cells plays a major role in the understanding of carcinogenic processes and targeted therapies (Nelson et al., 1999; Locker et al., 2006) .

In this regards, abnormal methylation patterns can also indirectly affect gene activity through disruption of transcription process or increasing the probability of mutational events resulted in chromosomal instability (Wajed et al., 2001; Ehrlich, 2002; Suzuki et al., 2006; Asiaf et al., 2014). Although global hypomethylation is commonly observed in malignant cells, the methylation profile alterations in cancer are mostly occur by hypermethylation changes in $\mathrm{CpG}$ islands of tumor suppressor genes (Jones and Baylin, 2007). Therefore, epigenetic alteration including promoter hypermethylation affects gene expression. According to UBE2Q1 and UBE2Q2 alteration in different cancers, it is supposed that the epigenetic mechanism could have an important regulatory role. However, epigenetic mechanisms that probably influence the expression of UBE2Q2 and its homologues, UBE2Q1, in malignancies are virtually unknown. There is no report about methylation pattern of these two genes promoters; therefore we decided to investigate the methylation status of UBE2Q1 and UBE2Q2 in CRC tumor samples and corresponding adjacent normal tissues as well as non- malignant control individuals.

\section{Materials and Methods}

\section{UBE2Q1 and UBE2Q2 genes annotation}

UBE2Q1 gene is located on chromosome 1q21 with an open reading frame (ORF) of 1269 base pairs belonging to the class II of human E2 ubiquitin conjugating enzymes (EC 6.3.2.19). A homologue of this gene, UBE2Q2, is located on chromosome $15 \mathrm{q} 23$ with an open reading frame (ORF) of 1128 base pairs. (Seghatoleslam et al., 2006; Seghatoleslam et al., 2012b). According to bioinformatic analysis, both genes have special properties for epigenetic alteration in their promoter. They have $\mathrm{C}+\mathrm{Gs} /$ total bases ratio $>50 \%$ and $\mathrm{CpG}$ observed/CpG expected $>0.6$. In addition, one $\mathrm{CpG}$ island was found in their promoters according to methyl primer express software (V.1.0 exe) calculation.

\section{Population study and tumor samples}

A total of $60 \mathrm{CRC}$ tumor samples as well as corresponding normal mucosa were collected from Iranian Table 1. Primers sequences

\begin{tabular}{lll}
\hline Gene & \multicolumn{1}{c}{ Sense sequence $\left(5^{\prime}\right.$-3’’) } & \multicolumn{1}{c}{ Antisense sequence(5’-3’) } \\
\hline UBE2Q1 Unmeth & TAGGGGTAGTAGTAGTTGGGGTT & CACTAACAATACAAAAACACTCA \\
UBE2Q1 Meth & GGGTAGTAGTAGTCGGGGTC & GCTAACAATACGAAAACGCT \\
UBE2Q2 Unmeth & TAG TTA TGA GGG GGG AGT TT & ACC ATT CCA CTT ACC AAC C \\
UBE2Q2 Meth & GTT ACG AGG GAG TTC & CGT TCC ACT CGA CCT A \\
\hline
\end{tabular}

patients attending Faghihi and Motahari hospitals during 2009 - 2011. 20 age and sex matched healthy participants were also selected from 50 volunteers who performed colonoscopy and had normal colonic mucosa. Patients were undergoing surgical procedure as a treatment and had not received any medication and radiotherapy before surgery. All samples were evaluated and subjected to histological diagnosis by an expert pathologist who also selected representative tissue sections for DNA extraction, and further molecular analyses. The splenic flexure was used as the anatomical boundary to define proximal and distal CRC. Sociodemographic characteristics such as age and gender were obtained by completion of a detailed questionnaire. Individuals with eligible criteria who provided written informed consent were enrolled. Ethics committee and institutional review board of Shiraz University of Medical Sciences approved the study. All Fresh samples were snap-frozen and stored at $-70^{\circ} \mathrm{C}$ until processing.

\section{DNA extraction \& Bisulfite modification}

DNAs were extracted from tumor and non-malignant samples according to the standard phenol/chloroform method (Mokarram et al., 2008). We determined UBE2Q1 and UBE2Q2 promoter methylation status by chemical treatment with sodium bisulfite and subsequent MSP, as described (Herman et al., 1996). In brief, this technique uses bisulfite modification to convert unmethylated, but not methylated, cytosine to uracil. MSP utilizes this difference to amplify specifically either methylated or unmethylated DNA.

\section{Methylation-specific PCR}

The sequences of the primers used for amplification of the promoter regions of each gene are listed in Table 1. The MSP primers were designed using Methyl Express $($ ) software version 1.0. Hotstart Taq polymerase was used with initial activation and denaturation $95^{\circ} \mathrm{C}$ for $5 \mathrm{~min}$; 40 cycles consist of $95^{\circ} \mathrm{C}, 45 \mathrm{sec} ;\left[62^{\circ} \mathrm{C}\right.$ for UBE2Q1 and

Figure 1. Representative examples of MSP reactions for promoter methylation analysis of UBE2Q2 gene in primary CRC tumors. The presence of a visible PCR product in those lanes marked $U$ indicates the presence of unmethylated genes; the presence of a product in those lanes marked $M$ indicates the presence of methylated genes. Lane 1 indicates the $50 \mathrm{bp}$ DNA size marker. Universal methylated DNA (UMD) and unmethylated lymphocyte DNA was used as positive and negative controls

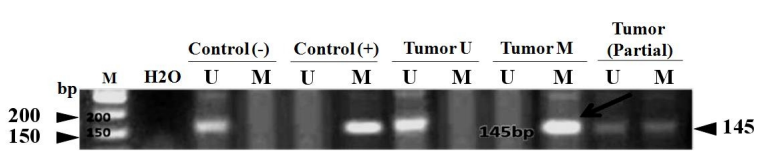


$45^{\circ} \mathrm{C}$ for UBE2Q2, $45 \mathrm{sec}$; ] and $72^{\circ} \mathrm{C}, 1$ min followed by final extension $72^{\circ} \mathrm{C}, 10 \mathrm{~min}$. In vitro methylated DNA and unmethylated lymphocytes DNA were used as positive and negative controls. MSP for UBE2Q1 and UBE2Q2 genes promoter were also performed in 40 and 60 modified DNA samples from patients with cancer as well as 20 nonmalignant samples, respectively. Representative example of MS-PCR assay has been shown in Figure 1.

\section{Statistical analysis}

The age of patients was a continuous variable, while race, gender, tumor location, differentiation, stage, genes methylation were categorical variables. The distribution of categorical variables was shown by frequency table and age by mean $( \pm \mathrm{SD})$. Associations between methylation of promoter methylation status with age, gender, differentiation, stages and tumor location were evaluated

Table 2. Clinical and Demographical Characteristics of the Patients

\begin{tabular}{lc}
\hline Number of patients & 60 \\
Age; Mean(range) & $61.6(36-81)$ \\
Gender & \\
$\quad$ Female & $22(36.7 \%)$ \\
$\quad$ Male & $38(63.3 \%)$ \\
Site & \\
$\quad$ Distal & $44(73.3 \%)$ \\
$\quad$ Proximal & $16(26.7 \%)$ \\
Age & \\
$\quad<60$ & $30(50 \%)$ \\
$\geq 60$ & $30(50 \%)$ \\
Differentiation & \\
Poor & $3(5 \%)$ \\
Moderate & $30(50 \%)$ \\
Well & $27(45 \%)$ \\
Stage & \\
1 & $11(18.3 \%)$ \\
2 & $35(58.3 \%)$ \\
3,4 & $14(23.3 \%)$ \\
\hline
\end{tabular}

using a chi square test. All analyses were performed using SPSS 18 software.

\section{Results}

Clinicopathological characteristics of patients

Selected characteristics of the study population are presented in Table 2. As shown in Table 2, 44 out of 60 tumor samples were distal $(73.3 \%)$. The majority of the tumors was at advanced stages and moderate /well differentiated histological grades, respectively. (81.6\% of cases were categorized in stage II and higher stage; $95 \%$ of cases were classified at moderate/well grades). In addition, 20 age matched control were also selected as non-malignant controls.

\section{$U B E 2 Q 1$ and $U B E 2 Q 2$ methylation status}

Although there were no significant differences between methylation pattern in the non malignant and adjacent normal tissues for both genes, but UBE2Q1 was found to be more methylated $(82.5 \%)$ in tumor tissues compared to 20 non-malignant tissues ( $n=20,100 \%$ unmethylated). In contrast, UBE2Q2 gene promoter was more unmethylated $(87.4 \%)$ in the majority of the tumor samples (Table 3 ). In addition, our results showed different patterns of UBE2Q2 methylation in non-malignant control tissues (16 out of 20 , ie. $80 \%$ were methylated).

\section{Age, sex and gene methylation}

Methylation status of UBE2Q1 and UBE2Q2 genes do not seem to be specifically associated with any age and sex parameters considered in this study. None of the two genes showed any age and sex-dependent methylation profile (Table 3).

\section{Tumor location and gene methylation}

According to Table 3, UBE2Q1 displayed higher methylation levels in distal tumors than in proximal ones, while UBE2Q2 gene showed less methylation

Table 3. Stratification analysis of tumors and UBE2Q1, UBE2Q2 genes promoter methylation frequency

\begin{tabular}{|c|c|c|c|c|c|c|}
\hline & \multicolumn{3}{|c|}{ UBE2Q1 } & \multicolumn{3}{|c|}{ UBE2Q2 } \\
\hline & $\mathrm{U}$ & M & $P$ value & $\mathrm{U}$ & M & $\mathrm{P}$ value \\
\hline \multicolumn{7}{|l|}{ Gender } \\
\hline Male & $5(12.5 \%)$ & $22(55 \%)$ & 0.12 & $30(48 \%)$ & $8(14 \%)$ & 0.1 \\
\hline Female & $2(5 \%)$ & $11(27.5 \%)$ & $17(39.4 \%)$ & $5(10 \%)$ & & \\
\hline Total & 0.175 & 0.825 & $8(7.4 \%)$ & 0.126 & & \\
\hline \multicolumn{7}{|l|}{ Age } \\
\hline$>60$ & $7(17.5 \%)$ & $11(27.5 \%)$ & 0.37 & $21(35 \%)$ & $9(15 \%)$ & 0.43 \\
\hline$\leq 60$ & $6(15 \%)$ & $16(40 \%)$ & & $25(41.6 \%)$ & $5(8.3)$ & \\
\hline \multicolumn{7}{|l|}{ Tumor location } \\
\hline Proximal & $2(5 \%)$ & $8(17.5 \%)$ & 0.03 & $10(16 \%)$ & $4(6.4 \%)$ & 0.02 \\
\hline Distal & $5(15 \%)$ & $25(62.5 \%)$ & $37(57.6 \%)$ & $9(16 \%)$ & & \\
\hline \multicolumn{7}{|l|}{ Diffrentiation } \\
\hline poor & 0 & 0 & 0 & $2(3.2 \%)$ & $1(1.6 \%)$ & 0 \\
\hline moderate & $4(10 \%)$ & $15(37.5 \%)$ & $24(37.5 \%)$ & $6(10 \%)$ & & \\
\hline well & $3(7.5 \%)$ & $18(45 \%)$ & $21(35 \%)$ & $6(10 \%)$ & & \\
\hline \multicolumn{7}{|l|}{ Stage } \\
\hline 1 & $2(5 \%)$ & $6(15 \%)$ & 0.02 & $7(10 \%)$ & $4(8 \%)$ & 0 \\
\hline 2 & $3(7.5 \%)$ & $17(42.5)$ & $28(43.2 \%)$ & $6(12.8 \%)$ & & \\
\hline 3,4 & $2(5 \%)$ & $10(25 \%)$ & $12(16 \%)$ & $3(6.4 \%)$ & & \\
\hline
\end{tabular}


levels in distal tumors. There were different methylation frequencies of UBE2Q1 and UBE2Q2 in proximal tumors versus distal tumors. $(\mathrm{p}<0.02)$ (Table 3$)$

\section{Tumor differentiation/ tumor stage and gene methylation}

UBE2Q1 and UBE2Q2 genes displayed different methylation profiles at differentiation and tumor stage status. UBE2Q1 gene displayed higher methylation in well and moderate differentiation levels and stage 2, 3, 4. In contrast, UBE2Q2 gene showed hypomethylated promoter profile in moderate and well differentiated tumor tissues and stage 2,3,4 (Table 3).

\section{Discussion}

In this study we showed the promoter methylation status of the two novel human genes, UBE2Q1 and UBE2Q2, for the first time. We explore that the methylation pattern for these two genes are different in the colon cancerous and non-malignant tissues. These findings point to their importance in colon tumorigenesis as a tumor suppressor and/or oncogene candidate.

Very little is known about the epigenetic mechanisms which regulate UBE2Q1 and UBE2Q2 expression in the non-malignant and cancerous tissues. But the presence of $\mathrm{CpG}$ Island in the promoter region of these two new human genes, as hall marker for epigenetic changes, probably influences their expression.

More than 1 million individuals are being diagnosed with colorectal cancer annually with different genetic or epigenetic changes in multiple genes. There are increasing numbers of genes that have been identified and are associated with colorectal cancer (Nelson et al., 1999; Weitz et al., 2005). Despite of developments in molecular mechanism of colorectal cancer understanding, identification of novel genes as molecular biomarker is still remarkable. There are some markers for colon cancer but have disadvantages due to low sensitivity or specificity (Shah et al., 2014). Recently, several researchers suggest that it may exert an important correlation between Ubiquitin-Conjugating enzyme family and cancer progression. Some of these studies have examined the important role of the aberrant expression of UBE2Q1 and UBE2Q2 in tumoregenesis (Seghatoleslam et al., 2006; Nikseresht et al., 2010; Seghatoleslam et al., 2012a; Seghatoleslam et al., 2012b; Shafiee et al., 2013; Seghatoleslam et al., 2014; Shafiee et al., 2014) but the mechanisms that control their expression remained uninvestigated.

The molecular defects in CRC can also be due to the changes that result in increased activity of oncogenes or loss of function of the tumor-suppressor genes. In this regard some studies have shown that the mutations in $\mathrm{K}$ - ras oncogene as well as APC, p53 and SMAD4/ DPC4 tumor-suppressor genes have occurred in colorectal cancer. These proteins with essential role in CRC are shown to be regulated by the UPS (Voutsadakis, 2008). UPS also plays a critical role in the availability of both transcription factors $\beta$-catenin and the tumor suppressor genes or oncogenes that mediate proliferation and differentiation in colorectal epithelium; therefore, defects in our two interested genes, as members of UPS, might accelerate carcinogenesis especially in colorectal cancer.

Other studies have also shown that UPS is strongly associated with the cell cycle events which control cell cycle progression (Dapas et al., 2003; Lamberti et al., 2004; Chuang et al., 2005). Therefore, UPS plays critical roles in control of various cellular proteins function including critical regulators of signal transduction, cell proliferation, and apoptosis for tumorigenesis (Arvand et al., 1998; Chen et al., 2002; Urano et al., 2002). A variety of E2 proteins have shown to be closely linked to the cell cycle progression and tumorigenesis. The role of two E2 enzymes, UbcH10 (also known as UBE2C or Ubc4) and UBE2S (also called E2-EPF) which are both working with E3 ligase APC /C (Anaphase Promoting Complex/ Cyclosome) in the regulation of cell cycle have been demonstrated (Bremm and Komander, 2011) UbcH10 plays a role in cell cycle progression and checkpoint control.

According to another studies, UBE2Q1 and UBE2Q2, as the putative E2 ubiquitin conjugating enzymes, may have remarkable roles in tumorigenesis via ubiquitination on the cell cycle progression components (Burger and Seth, 2004). UBE2Q2, as more studied gene than UBE2Q1, plays an important role in the cellular response to microtubule inhibition. Its inactivation also causes cells to undergo prophase arrest and apoptosis in $\mathbf{M}$ phase. These data suggesting that it might promote the development of aneuploidy or malignancy as an oncogene in M phase (Banerjee et al., 2007). UBE2Q2 participates in the regulation of cell cycle and check point control and is upregulated in a variety of solid tumors and hematological malignancies (Seghatoleslam et al., 2006; Seghatoleslam et al., 2012a; Seghatoleslam et al., 2012b; Shafiee et al., 2013; Chang et al., 2014; Seghatoleslam et al., 2014; Shafiee et al., 2014). In this regards, our finding based on un-methylated form of UBE2Q2 in tumor tissues confirmed that the overexpression of UBE2Q2 (Urano et al., 2002) might occur in cancer via epigenetic alteration and promote the development of malignancy. UBE2Q2 also showed high level of un-methylated allele in tumor tissue especially in distal tumors $(n=37,57.6 \%)$.

Epigenetic analysis of tumor cells plays a major role in the understanding of carcinogenic processes. In addition, epigenetic and mutation study led to the identification of silenced promoters. Therefore, according to UBE2Q2 methylation status in non-malignant control tissues which is hypermetylated and no methylated alleles were shown during cancer progression in tumor as well as normal adjacent tissues, we can strongly introduce it as oncogene during initiation and cancer progression process. In addition, our samples were also more hypomethylated in higher stages. Therefore, UBE2Q2 might act as an oncogene during the CRC cancer progression. Notably, the presence of UBE2Q2 un-methylated allele in CRC might demonstrate the oncogenic role of this gene, which is in agreement with the previous studies showing it's over expression during cancer progression in several cancers (Seghatoleslam et al., 2012a; Chang et al., 2014).

We also evaluated the methylation status of UBE2Q1 gene in CRC. As demonstrated its methylation frequency 
is different from its homologe, UBE2Q2 gene. UBE2Q1 was more methylated $(82.5 \%)$ in the tumor samples as well as normal adjacent tissues compared to the nonmalignant individuals. Hence, UBE2Q1 hypermethylation in colon cancer causes its inactivation which might lead to malignancy. Methylated form of UBE2Q1 in cancer will highlight an influence on cancer progression via epigenetic mechanism as tumor suppressor gene. Previous research also has shown UBE2Q1 down regulation in ALL (Seghatoleslam et al., 2012a). Another study revealed that inhibition of P53 pathway could decrease UBE2Q1 expression in apoptotic multiple myeloma cells (Wan et al., 2014).

So the methylation pattern of UBE2Q1 and UBE2Q2 has an influence in the process of cancer initiation and progression. On the other hand, since we also detected methylation alteration in normal adjacent tumor samples and in the samples of higher stages patients; we conclude that the epigenetic alteration of these two genes might act as tumor suppressor gene or oncogene during cancer progression. However, further investigation needs to have better conclusion about these differences because a limited statistical population were analyzed in our study. In addition, the precise amount of methylation found in our study should be evaluated in other population with the racial differences and more tumor samples with different stages and grades..

We have shown the promoter methylation pattern of the two novel human genes, UBE2Q1 and UBE2Q2, are different in colon cancer tissues. According to our finding it seems that inactivation of UBE2Q1 by hypermethylation as tumor suppressor gene and activation of UBE2Q2 by hypomethylation as oncogene might be specific role in CRC progression which could introduce them as new markers in CRC progression. In addition, probable epigenetic mechanisms of UBE2Q1 and UBE2Q2 genes in $\mathrm{CRC}$ carcinogenesis should be evaluated in the future to specify which mechanisms control the essential role of UBE2Q1 and UBE2Q2 in malignancies. It may include the related enzymes involved in methylation process such as DNMT3a, DNMT3b and HDAC and modification of histones and micro RNAs in more samples, with different stages and tumor location.

\section{Acknowledgements}

This article was extracted from the MSc thesis written by Fatemeh Shakiba-Jam and financially supported by Shiraz University of Medical sciences, Grant no. 91-6419.

\section{References}

Arvand A, Bastians H, Welford SM, et al (1998). EWS/FLI1 up regulates $\mathrm{mE2}-\mathrm{C}$, a cyclin-selective ubiquitin conjugating enzyme involved in cyclin B destruction. Oncogene, 17, 2039-45.

Asiaf A,Ahmad St, Fau - Aziz SA, et al (2014). Loss of expression and aberrant methylation of the $\mathrm{CDH} 1$ (E-cadherin) gene in breast cancer patients from Kashmir. Asian Pac J Cancer Prev, 15, 6397-403.

Atefeh Seghatoleslam AZ (2009). Effects of over-expression of loc92912 gene on cell cycle progression. IJMS, 34, 277-84.

Banerjee S, Brooks Ws Fau - Crawford DF, Crawford DF (2007). Inactivation of the ubiquitin conjugating enzyme UBE2Q2 causes a prophase arrest and enhanced apoptosis in response to microtubule inhibiting agents. Oncogene, 4, 6509-17.

Bassermann F, Pagano M (2010). Dissecting the role of ubiquitylation in the DNA damage response checkpoint in G2. Cell Death Differ, 17, 78-85.

Bremm A, Komander D (2011). Emerging roles for Lys11-linked polyubiquitin in cellular regulation. Trends Biochem Sci, 36, 355-63.

Burger AM, Seth AK (2004). The ubiquitin-mediated protein degradation pathway in cancer: therapeutic implications. Eur J Cancer, 40, 2217-29.

Chang R, Wei L, Lu Y, et al (2014). Upregulated expression of ubiquitin-conjugating enzyme E2Q1 (UBE2Q1) is associated with enhanced cell proliferation and poor prognosis in human hapatocellular carcinoma. J Mol Histol, 46, 45-56.

Chen A, Kleiman FE, Manley JL, et al (2002). Autoubiquitination of the BRCA1*BARD1 RING ubiquitin ligase.J Biol Chem, 277, 22085-92.

Chen L, Madura K (2005). Increased proteasome activity, ubiquitin-conjugating enzymes, and eEF1A translation factor detected in breast cancer tissue. Cancer Res, 65, 5599-606.

Cheng YW, Shawber C, Fau - Notterman D, et al (2006). Multiplexed profiling of candidate genes for $\mathrm{CpG}$ island methylation status using a flexible PCR/LDR/Universal Array assay. Genome Res, 16, 282-9.

Chuang SM, Chen L, Lambertson D, et al (2005). Proteasomemediated degradation of cotranslationally damaged proteins involves translation elongation factor 1A. Mol Cell Biol, 25, 403-13.

Dapas B, Tell G, Scaloni A, et al (2003). Identification of different isoforms of eEF1A in the nuclear fraction of human T-lymphoblastic cancer cell line specifically binding to aptameric cytotoxic GT oligomers. Eur J Biochem, 270, 3251-62.

Ehrlich M (2002). DNA methylation in cancer: too much, but also too little. Oncogene, 12, 5400-13.

Herman JG, Graff JR, Myohanen S, et al (1996). Methylationspecific PCR: a novel PCR assay for methylation status of CpG islands. Proc Natl Acad Sci U S A, 93, 9821-6.

Jones PA, Baylin SB (2007). The epigenomics of cancer. Cell, 128, 683-92.

Lamberti A, Caraglia M, Longo O, et al (2004). The translation elongation factor $1 \mathrm{~A}$ in tumorigenesis, signal transduction and apoptosis: review article. Amino Acids, 26, 443-8.

Locker GY, Hamilton S, Harris J, et al (2006). ASCO 2006 update of recommendations for the use of tumor markers in gastrointestinal cancer. J Clin Oncol, 24, 5313-27.

Maeda H, Miyajima N, Fau - Kano S, et al (2009). Ubiquitinconjugating enzyme UBE2Q2 suppresses cell proliferation and is down-regulated in recurrent head and neck cancer. Mol Cancer Res, 7, 1553-62.

Mokarram P, Naghibalhossaini F, Fau - Saberi Firoozi M, et al (2008). Methylenetetrahydrofolate reductase C677T genotype affects promoter methylation of tumor-specific genes in sporadic colorectal cancer through an interaction with folate/vitamin B12 status. World J Gastroentrol, 14, 3662-71.

Nelson RL, Persky V, Turyk M (1999). Determination of factors responsible for the declining incidence of colorectal cancer. Dis Colon Rectum, 42, 741-52.

Nikseresht M, Seghatoleslam A, Fau - Monabati A, et al (2010). Overexpression of the novel human gene, UBE2Q2, in breast cancer. Cancer Genet Cytogenet, 197, 101-6.

Seghatoleslam A, Bozorg-Ghalati F, Monabati A, et al (2014). 


\section{Pooneh Mokarram et al}

UBE2Q1, as a Down Regulated Gene in Pediatric Acute Lymphoblastic Leukemia. int j mol cell med, 3, 95-101.

Seghatoleslam A, Monabati A, Fau - Bozorg-Ghalati F, et al (2012a). Expression of UBE2Q2, a putative member of the ubiquitin-conjugating enzyme family in pediatric acute lymphoblastic leukemia. Arch Iran Med, 15, 352-5.

Seghatoleslam A, Nikseresht M, Fau - Shafiee SM, et al (2012b). Expression of the novel human gene, UBE2Q1, in breast tumors. Mol Biol Rep, 39.

Seghatoleslam A, Zambrano A, Fau - Millon R, et al (2006). Analysis of a novel human gene, LOC92912, over-expressed in hypopharyngeal tumours. Biochem Biophys Acta, 6.

Shafiee SM, Seghatoleslam A, Nikseresht M, et al (2013). UBE2Q1 expression in human colorectal tumors and cell lines. Mol Biol Rep, 40, 7045-51.

Shafiee SM, Seghatoleslam A, Nikseresht M, et al (2014). Expression Status of UBE2Q2 in Colorectal Primary Tumors and Cell Lines. Iran J Med Sci, 39, 196-202.

Shah R, Jones E, Vidart V, et al (2014). Biomarkers for early detection of colorectal cancer and polyps: systematic review. Cancer Epidemiol Biomarkers Prev, 23, 1712-28.

Srivastava S, Shahi UP, Dibya A, et al (2014). Distribution of HPV genotypes and involvement of risk factors in cervical lesions and invasive cervical cancer: a study in an indian population. Int J Mol Cell Med, 3, 61-73.

Suzuki H, Toyota M, Fau - Sato H, et al (2006). Roles and causes of abnormal DNA methylation in gastrointestinal cancers. Asian Pac J Cancer Prev, 7, 177-85.

Urano T, Saito T, Tsukui T, et al (2002). Efp targets 14-3-3 sigma for proteolysis and promotes breast tumour growth. Nature, 417, 871-5.

van Wijk SJ, Timmers HT (2010). The family of ubiquitinconjugating enzymes (E2s): deciding between life and death of proteins. Faseb J, 24, 981-93.

Voutsadakis IA (2008). The ubiquitin-proteasome system in colorectal cancer. Biochem Biophys Acta, 1782, 800-8.

Wajed SA, Laird PW, DeMeester TR (2001). DNA methylation: an alternative pathway to cancer. Ann Surg, 234, 10-20.

Wan C, Chen J, Fau - Hu B, et al (2014). Downregulation of UBE2Q1 is associated with neuronal apoptosis in rat brain cortex following traumatic brain injury. J Neurosci Res, 92, 1-12.

Weitz J, Koch M, Debus J, et al (2005). Colorectal cancer. Lancet, 365, 153-65.

Zhu S, Yao F, Fau - Li WH, et al (2013). PKC?-dependent activation of the ubiquitin proteasome system is responsible for high glucose-induced human breast cancer MCF-7 cell proliferation, migration and invasion. Asian Pac J Cancer Prev, 14. 